PASJ: Publ. Astron. Soc. Japan , 1-??,

(C) 2018. Astronomical Society of Japan.

\title{
Spectropolarimetry of the superwind filaments of the starburst galaxy M82: kinematics of dust outflow
}

\author{
Michitoshi Yoshida ${ }^{1,2} *$, Koji, S. Kawabata ${ }^{1}$ and Youichi OнуамA ${ }^{3}$ \\ ${ }^{1}$ Hiroshima Astrophysical Science Center, Hiroshima University, Higashi-Hiroshima, Hiroshima 739-8526, Japan. \\ yoshidam@hiroshima-u.ac.jp \\ ${ }^{2}$ Okayama Astrophysical Observatory, National Astronomical Observatory of Japan, Kamogata, Asakuchi, Okayama 719-0232, \\ Japan \\ ${ }^{3}$ Academia Sinica, Institute of Astronomy and Astrophysics, P.O. Box 23-141, Taipei 10617, Taiwan, R.O.C.
}

(Received ; accepted )

\begin{abstract}
Spectropolarimetry results for the starburst galaxy M82 are presented. The optical emission lines of the filaments in the energetic outflow ("superwind") from the nuclear starburst region of M82 are substantially polarized. The $\mathrm{H} \alpha$ polarization degrees and angles measured by our study are consistent with previous narrowband imaging polarimetry data. The polarized emission lines are redshifted with respect to the emission lines in the total light and systemic motion of the galaxy. The emission line intensity ratios $[\mathrm{N} \mathrm{II}] / \mathrm{H} \alpha$ and $[\mathrm{S} \mathrm{II}] / \mathrm{H} \alpha$ in the polarized light are similar to those of the nuclear star-forming region. In addition, the electron density $N_{\mathrm{e}}$ derived from the $[\mathrm{S} \mathrm{II}] \lambda 6731 / \lambda 6717$ line ratio of the polarized light is $\sim 600-1000 \mathrm{~cm}^{-3}$ at a distance of more than $1 \mathrm{kpc}$ from the nucleus, whereas the $N_{\mathrm{e}}$ derived from the total light are less than $300 \mathrm{~cm}^{-3}$. These facts strongly suggest that the emission from the nuclear starburst of M82 is scattered by dust grains entrained and transported outward by the superwind. A simple hollow biconical outflow model shows that the velocity of the outflowing dust grains, $v_{\mathrm{d}}$, ranges from 100 to 200 $\mathrm{km} \mathrm{s}^{-1}$ near the nucleus, decreases monotonically with the distance from the nucleus, and reaches $\sim 10$ $\mathrm{km} \mathrm{s}^{-1}$ at around $1 \mathrm{kpc}$. The motion of the dust is substantially slower than that of both ionized gas $\left(v_{\mathrm{H} \alpha} \sim 600 \mathrm{~km} \mathrm{~s}^{-1}\right)$ and molecular gas $\left(v_{\mathrm{CO}} \sim 200 \mathrm{~km} \mathrm{~s}^{-1}\right)$ at the same distance from the nucleus of M82. This indicates that dust grains in the superwind are kinematically decoupled from both gas components at large radii. Since the dust velocity $v_{\mathrm{d}}$ is much less than the escape velocity of M82 $\left(v_{\mathrm{esc}} \approx 170 \mathrm{~km} \mathrm{~s}^{-1}\right.$ at $1.5 \mathrm{kpc}$ from the nucleus), most of the dust entrained by the superwind cannot escape to intergalactic space, and may fall back into the galaxy disk without any additional acceleration mechanisms (such as radiation pressure).
\end{abstract}

Key words: galaxies:starburst — galaxies:nearby — galaxies:individual(M82) — interstellar medium:dust

\section{Introduction}

Active star formation in starburst galaxies plays one of the most important roles in galaxy evolution. In spite of its short duration $\left(\sim 10^{7-8}\right.$ yrs $)$, a starburst reforms a substantial amount of the interstellar medium (ISM) of a galaxy into stars. In addition, a starburst creates an enormous hot outflow from a galaxy via the collective effect of supernovae explosions and the stellar winds of massive stars. This energetic outflow, known as a "superwind," is a ubiquitous phenomenon in starburst galaxies (e.g., Heckman 2003; Veilleux et al. 2005). Theoretical studies estimate that the terminal velocity of the hot gas of a superwind reaches $\sim 10^{3} \mathrm{~km} \mathrm{~s}^{-1}$ (e.g Chevalier \& Clegg 1985), far exceeding the typical escape velocity of a galaxy. Metal-rich gas from the starburst region and ambient disk gas are expelled from the galaxy disk and pollute the galaxy halo and intergalactic space. Rapid * Based on data collected with the Subaru Telescope operated by
the National Astronomical Observatory of Japan consumption of the interstellar medium by a superwind will cause star formation in a galaxy to cease abruptly, although part of the expelled gas returns to the disk and induces further star formation. These negative and positive feedbacks greatly affect the chemical evolution of a galaxy.

A significant amount of dust is associated with superwinds. Submillimeter (sub-mm), far-infrared (IR), and mid-IR maps, optical color maps, and polarization studies of several nearby starburst galaxies have exhibited extended dust emission along the galaxy minor axis (Alton et al. 1999; Leeuw \& Robson 2009; Ichikawa et al. 1994; Scarrott et al. 1991; Engelbracht et al. 2006; Kaneda et al. 2010). Sub-mm observations of some starburst galaxies suggest that the mass of the dust may reach $\sim 10^{6-7}$ $\mathrm{M}_{\odot}$ (Alton et al. 1999; Leeuw \& Robson 2009). Although such a large-scale dust outflow has been inferred to play an important role in the evolution and metal enrichment of the intergalactic medium and halo gas, as well as the evolution of the host galaxy itself, the fate of this dust has thus far remained uncertain. This is because kinematic 
information on dust outflow is quite difficult to obtain, since dust produces no sharp emission or absorption lines by which its radial velocity can be measured.

One of the most promising techniques for probing the motion of dust in and around superwinds is optical spectropolarimetry. Outflowing dust grains entrained by a superwind scatter and polarize the continuum light and emission lines emanating from the nuclear starburst region. In other words, the dust grains act as "moving mirrors" for nuclear light. Hence, the velocity measured by the polarized emission lines of a superwind must reflect the motion of the dust with respect to the nucleus. Motivated by this idea, we carried out deep optical spectropolarimetry of the prototypical starburst galaxy M82 to reveal the dust kinematics of its superwind.

M82 is distinguished by its very bright, kpc-scale superwind (Nakai et al. 1987; Seaquist \& Clark 2001; Matsushita et al. 2005; Bierão et al. 2008; Bland \& Tully 1988; Shopbell \& Bland-Hawthorn 1998; Ohyama et al. 2002; Mutchler et al. 2007; Bregman et al. 1995; Tsuru et al. 2007; Ranalli et al. 2008; Strickland \& Heckman 2007). The superwind of M82 is accompanied by large extraplanar dust filaments, as well as hot ionized gas (Ichikawa et al. 1994; Alton et al. 1999; Thuma et al. 2000). Alton et al. (1999) found a huge dust envelope extending along the minor axis of M82 using the $850-\mu$ m sub-mm observation. Recently, Leeuw \& Robson (2009) detected a much fainter dust emission as far as $1.5 \mathrm{kpc}$ from the galaxy disk. They found that the sub-mm morphology has a north-south asymmetry, which is consistent with the $\mathrm{H} \alpha$ and X-ray morphologies. Mid- and far-IR maps recently obtained using IR space telescopes reveal a complex structure in the kpc-scale filaments of polycyclic aromatic hydrocarbon (PAH) dust extending along the minor axis of M82 (Engelbracht et al. 2006; Kaneda et al. 2010; Roussel et al. 2010). The highly polarized nature of the optical continuum and $\mathrm{H} \alpha$ emission of the outer region of M82 also indicates the presence of a vast quantity of dust in the superwind (Schmidt et al. 1976; Bingham et al. 1976; Visvanathan \& Sandage 1972; Scarrott et al. 1991). The polarization degree of the $\mathrm{H} \alpha$ emission reaches $30 \%$ in some areas, and the polarization angle is almost perpendicular to the radial direction drawn from the nucleus of the galaxy (Scarrott et al. 1991). Therefore, M82 is an ideal object for studying dust kinematics in a starburst superwind via optical spectropolarimetry.

In this paper, we present the results of deep optical spectropolarimetric observations of the superwind of M82. This is the first attempt to ascertain the spatial structure of the dust motion in a starburst superwind via spectropolarimetry. We adopted $3.89 \mathrm{Mpc}$ as the distance to M82 (Sakai \& Madore 1999), which yields a linear scale of 18.9 pc $\operatorname{arcsec}^{-1}$ for the galaxy.

\section{Observations}

Spectropolarimetric observations of M82 were made with FOCAS (Kashikawa et al. 2002), attached to the Cassegrain focus of the Subaru Telescope (Kaifu et al.

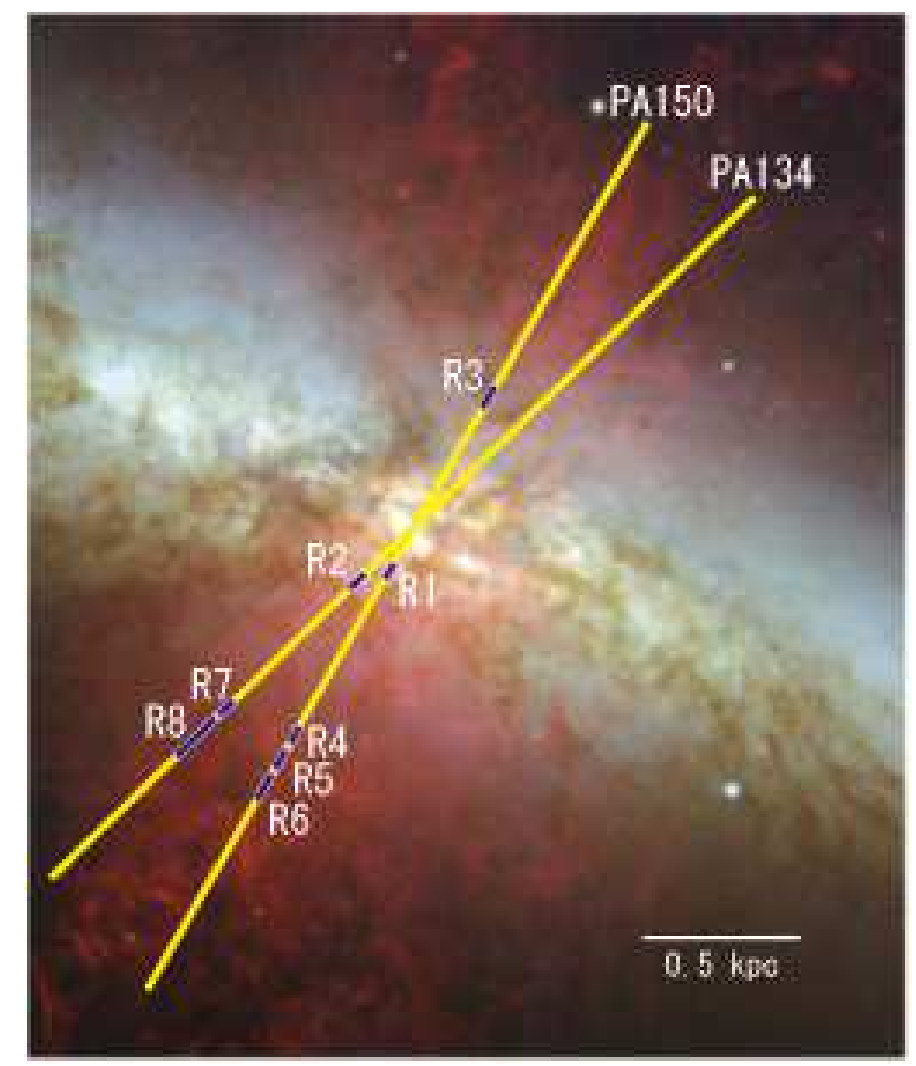

Fig. 1. The slit positions overlaid on a false color (blue: $V$, green: $R$, and red:H $\alpha$ ) image of M82. Regions discussed in the text are labeled $\mathrm{R} 1-\mathrm{R} 8$.

2000), on December 22 and 23, 2003. The observations were carried out using the spectropolarimetric mode of FOCAS (Kawabata et al. 2003). We used a slit mask with eight $0^{\prime \prime} .6$ (width) $\times 20^{\prime \prime} .6$ (length) slitlets at $23^{\prime \prime} .7$ intervals, and a VPH grism with 665 grooves $\mathrm{mm}^{-1}$ and a center wavelength of $6500 \AA$. The resultant spectral resolving power was $\lambda / \Delta \lambda \approx 1700$, determined by the combination of the slit and the grating. The separation direction of the beam splitter was perpendicular to the direction of the slit length, and spectra of both ordinary and extraordinary rays were obtained simultaneously.

The PA of the slit was set at $150^{\circ}$ and $134^{\circ}$. We placed the slit center at the position of the $2.2 \mu \mathrm{m} \mathrm{nu}-$ cleus (Telesco et al. 1991). A unit data set consists of exposures taken at four different position angles (PAs; $0^{\circ}$, $\left.22.5^{\circ}, 45^{\circ}, 67.5^{\circ}\right)$ of the half-wave plate. We took one data set with an exposure time of $600 \mathrm{~s}$ per exposure for PA $150^{\circ}$, and four data sets with an exposure time of $720 \mathrm{~s}$ per exposure for PA $134^{\circ}$. Thus, the total exposure times were $2400 \mathrm{~s}$ for PA $150^{\circ}$ and $11520 \mathrm{~s}$ for PA $134^{\circ}$. Figure 1 shows the positions of the slits overlaid on an image taken with FOCAS (Ohyama et al. 2002). The regions in which the spectra were extracted and summed are also shown in Figure 1.

We observed both an unpolarized star and a highly polarized star to calibrate the polarization. We also observed 

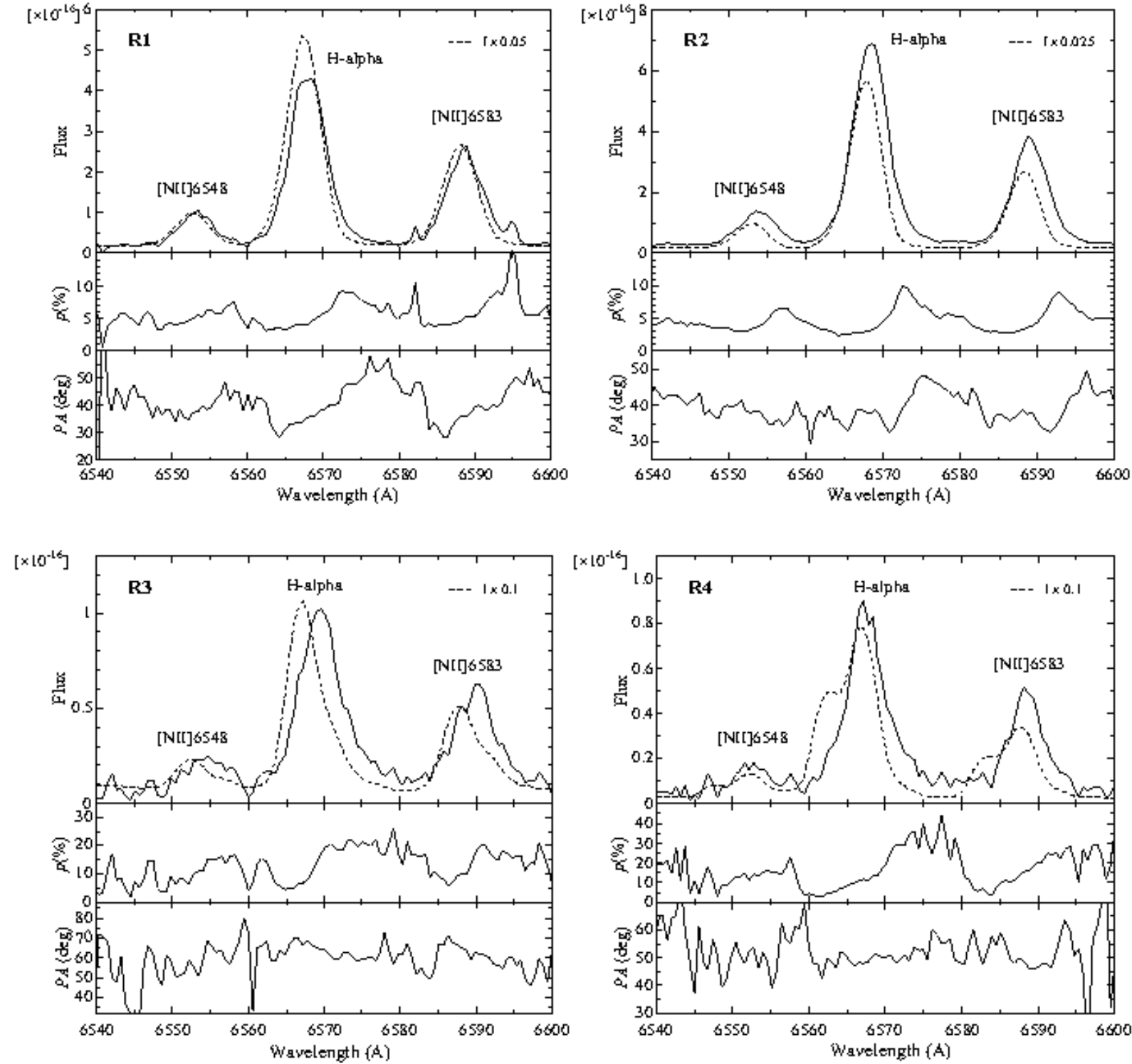

Fig. 2. Spectra of total light and polarized light around the $\mathrm{H} \alpha+[\mathrm{N} \mathrm{II}]$ wavelength region for $\mathrm{R} 1-\mathrm{R} 4$. The polarized spectra are represented by the solid line in the upper panels of each figure. The total light spectra are scaled by a factor shown in the upper-right corner of each figure and are represented by the dashed line. The polarization degree and polarization position angle are shown in the middle and bottom panels of each figure, respectively. 

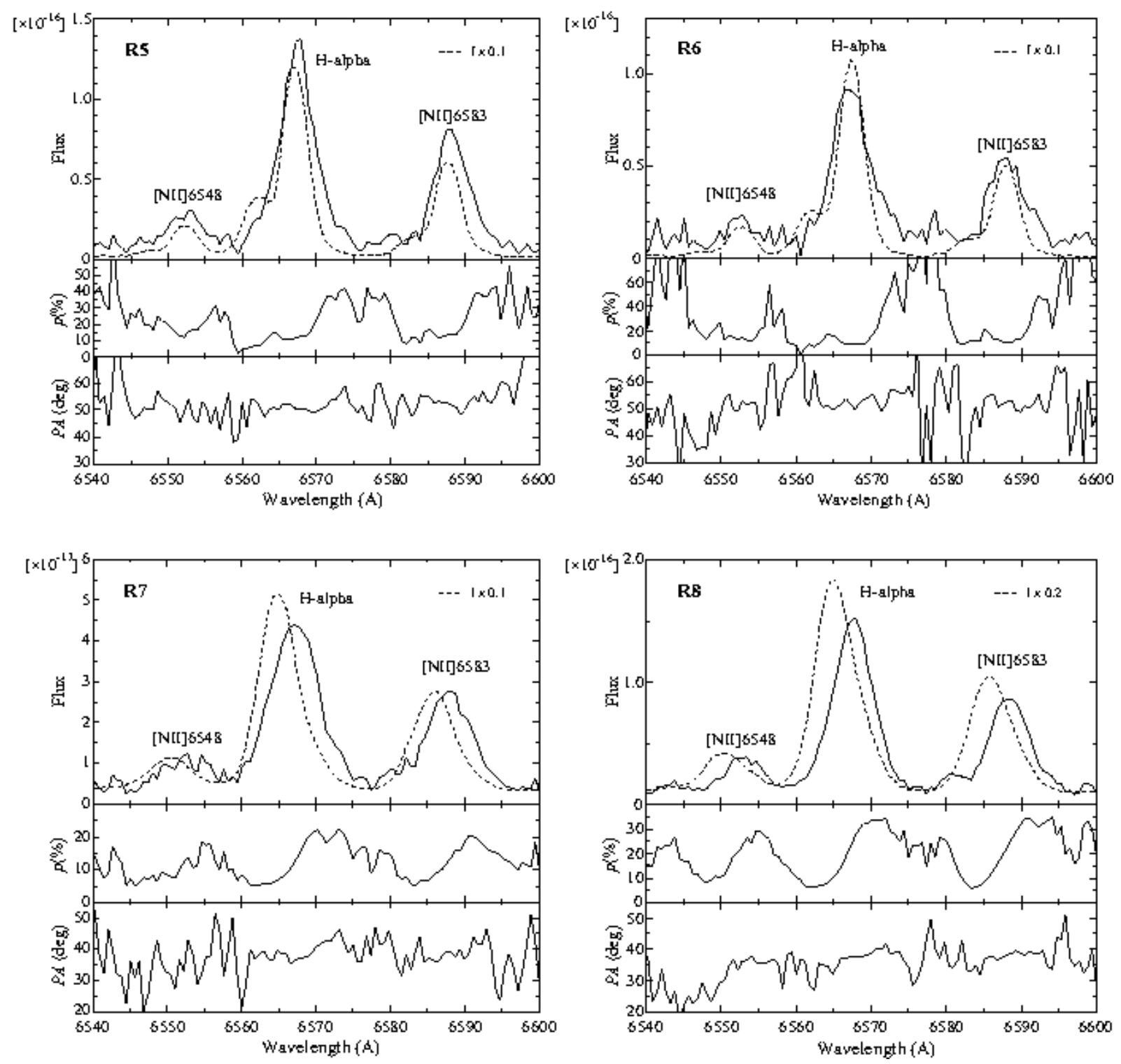

Fig. 3. Spectra around the $\mathrm{H} \alpha+[\mathrm{N}$ II] wavelength region for $\mathrm{R} 5-\mathrm{R} 8$. The layout is the same as in Figure 2 .

a spectrophotometric standard star. The first night of the observing run, December 22, was clear and photometric. The second night was not photometric, with some small passing clouds. The seeing was approximately $0^{\prime \prime} .6$ the first night and $0^{\prime \prime} .7-0^{\prime \prime} .8$ the second night.

\section{Data Reduction}

Standard CCD data reduction was applied to the raw CCD frames using IRAF. The bias level was subtracted from all frames. Since the dark current was negligible $(<1$ ADU), dark subtraction was not performed. Flat fielding was carried out using the dome flat data averaged over the four PAs of the half-wave plate.

We extracted one-dimensional spectra of ordinary and extraordinary rays in eight regions (R1-R8) of the twodimensional spectral images. The locations of $\mathrm{R} 1-\mathrm{R} 8$ are shown in Figure 1. The distances from the nucleus and the areas of the regions are summarized in Table 1. We estimated the sky background components from the twodimensional spectra in the outer region $\left(140-145^{\prime \prime}\right.$ from the nucleus), where no significant $\mathrm{H} \alpha$ flux was recorded. The polarization parameters were calculated from eight spectra (ordinary and extraordinary at the four waveplate PAs) employing the method described in $\$ 6.1 .2$ of Tinbergen (1996). Our observations of unpolarized stars indicated that instrumental polarization was negligible $(\lesssim 0.1 \%)$. Moreover, our measurements of flat-field lamps through fully polarizing filters showed that the depolarization factor was also negligible $(\lesssim 0.05)$. Therefore, we made no correction for instrumental polarization and depolarization. The zero point of the position angle on the sky was determined from the observation of the highly polarized star. 

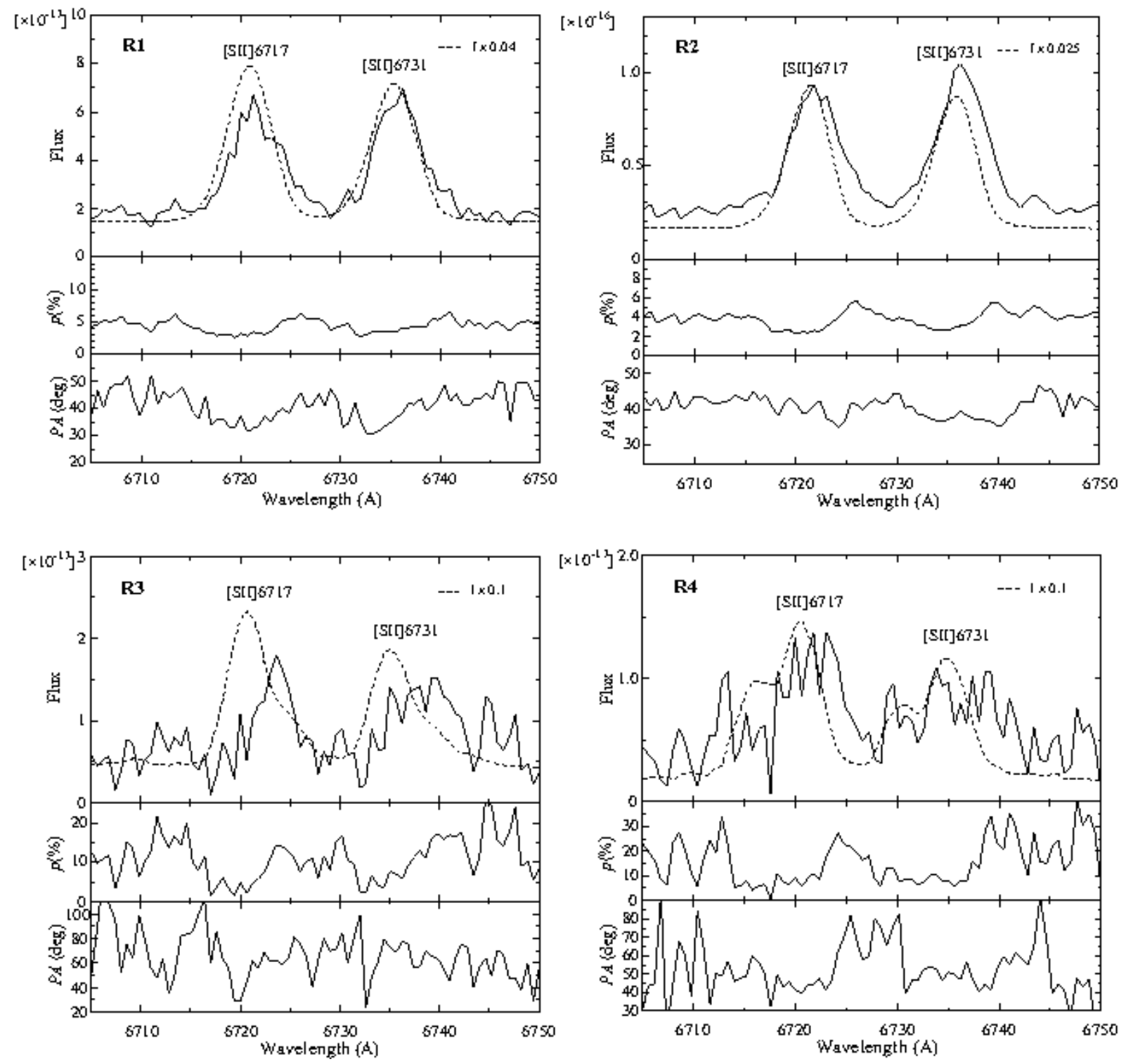

Fig. 4. Spectra around the $[\mathrm{S} \mathrm{II}]$ wavelength region for R1-R4. The layout is the same as in Figure 2.

We fitted Gaussian profiles to the emission line spectra for both the total light and the polarized light. First we applied Gaussian fitting to the $\mathrm{H} \alpha+[\mathrm{N}$ II] spectra, assuming that $\mathrm{H} \alpha,[\mathrm{N} \mathrm{II}] \lambda 6548$, and $[\mathrm{N}$ II] $] 6583$ have the same radial velocities and FWHMs. We fixed the emission line intensity ratio $[\mathrm{N} \mathrm{II}] \lambda 6583 / \lambda 6548$ at 3 for this procedure. The $\mathrm{H} \alpha+[\mathrm{N}$ II] emission lines of the total light in $\mathrm{R} 3, \mathrm{R} 7$, and $\mathrm{R} 8$ have a remarkable red-wing component, while those in R4, R5, and R6 exhibit a doublepeak profile. We fitted double Gaussian profiles to the $\mathrm{H} \alpha+[\mathrm{N} \mathrm{II}]$ lines of these regions to decompose their asymmetric profiles. The radial velocities and FWHMs of all components were assumed to be the same for the decomposition. We then applied Gaussian fitting to the [S II] $] \lambda 6717,6731$ lines, assuming their radial velocities and FWHMs to be the same as those of the $\mathrm{H} \alpha+[\mathrm{N}$ II] lines. Some asymmetric features were also found in the polarized emission line profiles of $\mathrm{R} 3-\mathrm{R} 7$, but the $\mathrm{S} / \mathrm{N}$ ratios were not high enough to obtain a reliable decomposition. Hence we applied a single Gaussian fitting to all the polarized emission lines, and then corrected for instrumental broadening by applying the following simple equation: $\mathrm{FWHM}=\sqrt{\mathrm{FWHM}_{\text {fit }}^{2}-\mathrm{FWHM}_{\mathrm{inst}}^{2}}$, where $\mathrm{FWHM}_{\text {fit }}$ and FWHM $_{\text {inst }}$ are the FWHM obtained from the fitting and that of the instrumental profile, respectively. We determined that $\mathrm{FWHM}_{\text {inst }}=140 \mathrm{~km} \mathrm{~s}^{-1}$ by fitting the sky emission lines near $\mathrm{H} \alpha$. The physical parameters derived by the above procedure are listed in Tables 1 and 2 .

\section{Properties of the polarized emission lines}

Figures 2 and 3 show the total fluxes, polarized fluxes, polarization degrees, and polarization angles of the $\mathrm{H} \alpha+[\mathrm{N}$ II] regions of the eight selected regions along the 

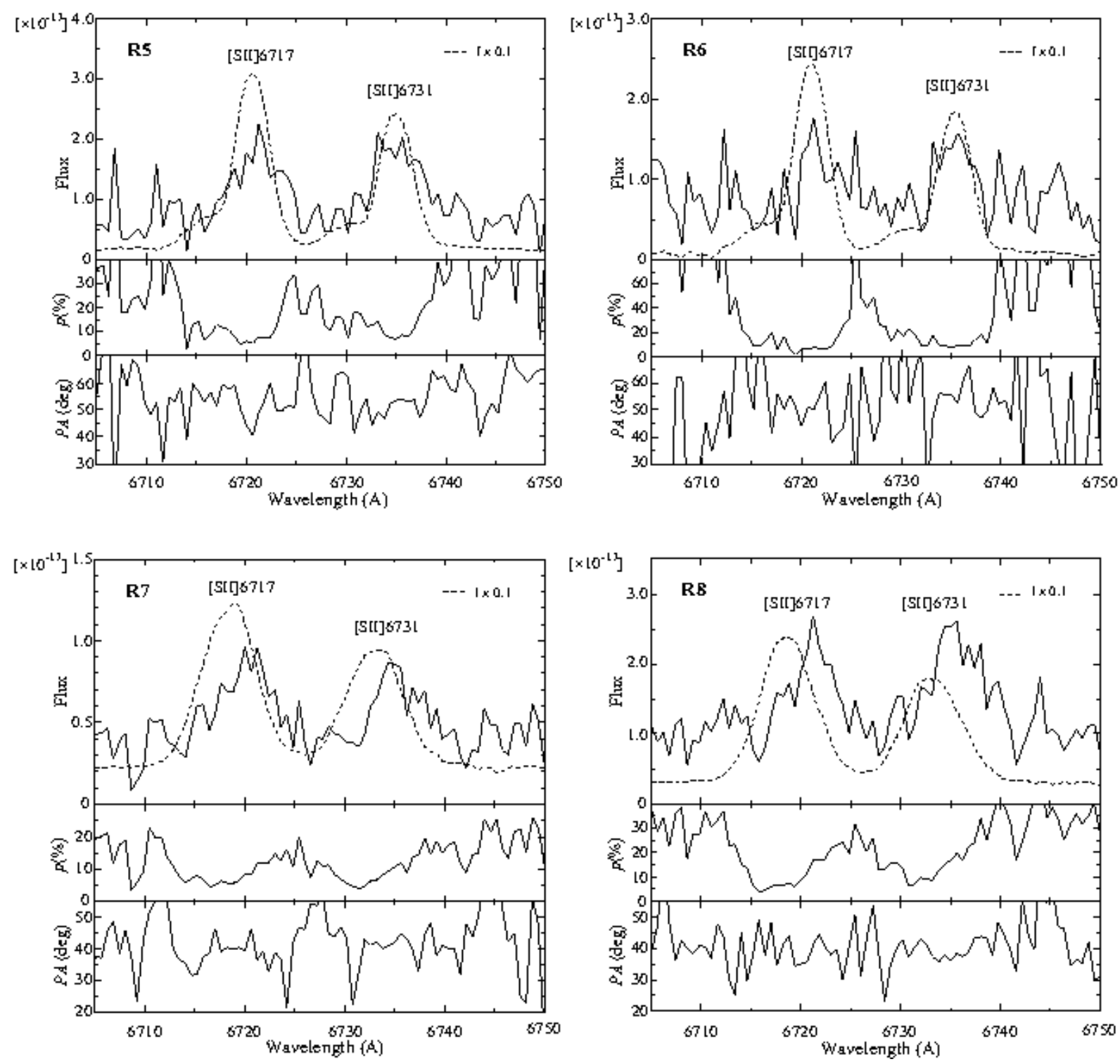

Fig. 5. Spectra around the $[\mathrm{S} \mathrm{II}]$ wavelength region for R5-R8. The layout is the same as in Figure 2.

slits (R1-R8). Figures 4 and 5 show the same data for the [S II] region of the spectra. We summarize the physical parameters derived from the total light spectra in Table 1. Since double Gaussian fittings were applied to the emission line profiles of $\mathrm{R} 3-\mathrm{R} 8$, the fitted parameters for each component are listed separately for these regions in Table 1. The emission line fluxes of the polarized $\mathrm{H} \alpha$, [N II $] \lambda 6581$ and $[\mathrm{S}$ II] $] \lambda \lambda 6717,6731$, the velocities and FWHMs of the polarized $\mathrm{H} \alpha$, and the electron densities $N_{\mathrm{e}}$ derived from the polarized [S II] line intensity ratios are given in Table 2. We assumed an electron temperature of $10^{4} \mathrm{~K}$ in calculating $N_{\mathrm{e}}$. The radial velocities are corrected to heliocentric values.

The intensity-weighted polarization degrees of the emission lines range from $5 \%$ to $15 \%$ (see Table 2). The polarization degree begins to decrease on the blue side of the emission lines, reaches its minimum near the center of the lines, increases again on the red side of the lines, and attains a peak at the red wings of the lines. Except for $\mathrm{R} 1$ and $\mathrm{R} 2$, the polarization angles are almost constant $\left(\approx 40^{\circ}-60^{\circ}\right)$ throughout the $\mathrm{H} \alpha+[\mathrm{N} \mathrm{II}]$ spectral region, and no difference exists between the polarization angles of the continuum and emission lines. In the spectra of $\mathrm{R} 1$ and $\mathrm{R} 2$, the polarization angles increase toward the red part of the emission lines (upper panels of Figure 2). The polarization angles are $\approx 35^{\circ}$ in the continuum and in the blue part of the emission lines, while the angles reach $\approx 50^{\circ}-60^{\circ}$ at the red wings of the lines. Except for the fine structures seen in R1 and R2, the polarization vectors are generally perpendicular to radial lines drawn from the M82 nucleus.

Figure 6 shows the radial velocities of the total light and polarized light versus the distance from the nucleus. Clearly the polarized emission line spectra are redshifted 
Table 1. Physical Parameters of the $\mathrm{H} \alpha$-emitting Gas in the M82 Superwind

\begin{tabular}{|c|c|c|c|c|c|c|c|c|c|c|}
\hline ID & $\begin{array}{l}\mathrm{PA}^{*} \\
(\mathrm{deg})\end{array}$ & $\begin{array}{l}\mathrm{D}^{\dagger} \\
(\mathrm{pc})\end{array}$ & $\begin{array}{c}\text { Area } \\
(\mathrm{pc} \times \mathrm{pc})\end{array}$ & $f_{\mathrm{H} \alpha}{ }^{\mp}$ & $\begin{array}{c}v \\
\left(\mathrm{~km} \mathrm{~s}^{-1}\right)\end{array}$ & $\begin{array}{c}\text { FWHM } \\
\left(\mathrm{km} \mathrm{s}^{-1}\right)\end{array}$ & 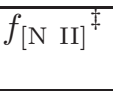 & $f_{\left[\begin{array}{ll}\mathrm{S} & \mathrm{II}\end{array}\right] 6717^{\ddagger}}$ & $\left.f_{[\mathrm{S}} \mathrm{III}\right] 6731^{\mp}$ & $\begin{array}{c}N_{\mathrm{e}, \mathrm{t}} \\
\left(\mathrm{cm}^{-3}\right)\end{array}$ \\
\hline $\mathrm{R} 1$ & 150 & 140 & $11 \times 28$ & 97 & $215 \pm 10$ & $189 \pm 22$ & 46 & 15 & 13 & $\overline{370 \pm 10}$ \\
\hline $\mathrm{R} 2$ & 134 & 245 & $11 \times 32$ & 190 & $234 \pm 10$ & $161 \pm 26$ & 85 & 26 & 25 & $450 \pm 10$ \\
\hline \multirow[t]{2}{*}{ R3 } & 150 & 630 & $11 \times 38$ & 7.5 & $192 \pm 11$ & $150 \pm 28$ & 3.3 & 1.4 & 1.1 & $120 \pm 10$ \\
\hline & & & & 2.3 & $385 \pm 12$ & $224 \pm 19$ & 1.4 & 0.4 & 0.4 & $370 \pm 10$ \\
\hline \multirow[t]{2}{*}{$\mathrm{R} 4$} & 150 & 735 & $11 \times 28$ & 6.7 & $195 \pm 11$ & $187 \pm 23$ & 2.9 & 1.1 & 0.85 & $110 \pm 10$ \\
\hline & & & & 2.6 & $-32 \pm 14$ & $75 \pm 63$ & 0.83 & 0.41 & 0.31 & $120 \pm 10$ \\
\hline \multirow{2}{*}{ R5 } & 150 & 805 & $11 \times 32$ & 8.7 & $205 \pm 11$ & $121 \pm 35$ & 4.4 & 2.1 & 1.6 & $120 \pm 10$ \\
\hline & & & & 2.4 & $-43 \pm 13$ & $95 \pm 46$ & 0.67 & 0.31 & 0.26 & $260 \pm 10$ \\
\hline \multirow[t]{2}{*}{ R6 } & 150 & 910 & $11 \times 43$ & 7.7 & $215 \pm 11$ & $116 \pm 37$ & 3.6 & 1.7 & 1.2 & $70 \pm 10$ \\
\hline & & & & 1.7 & $-40 \pm 13$ & $107 \pm 41$ & 0.57 & 0.24 & 0.21 & $320 \pm 10$ \\
\hline \multirow[t]{2}{*}{ R7 } & 134 & 840 & $11 \times 29$ & 3.0 & $83 \pm 10$ & $178 \pm 23$ & 1.2 & 0.75 & 0.50 & $<50$ \\
\hline & & & & 2.5 & $167 \pm 11$ & $325 \pm 12$ & 1.7 & 0.41 & 0.37 & $400 \pm 10$ \\
\hline \multirow[t]{2}{*}{$\mathrm{R} 8$} & 134 & 980 & $11 \times 77$ & 3.0 & $75 \pm 14$ & $130 \pm 33$ & 1.3 & 0.98 & 0.61 & $<50$ \\
\hline & & & & 7.0 & $155 \pm 11$ & $295 \pm 14$ & 4.3 & 1.4 & 1.2 & $260 \pm 10$ \\
\hline
\end{tabular}

* The position angle of the slit.

$\dagger$ The projected distance from the nucleus.

$\ddagger$ The emission line flux in units of $10^{-15} \mathrm{erg} \mathrm{s}^{-1} \mathrm{~cm}^{-2}$.

Table 2. Physical Parameters of the Gas Measured in Polarized Emission Lines of the M82 Superwind

\begin{tabular}{cccccccccc}
\hline ID & $\begin{array}{c}\text { P.Deg. } \\
(\%)\end{array}$ & $\begin{array}{c}\text { P.PA }^{\dagger} \\
(\mathrm{deg})\end{array}$ & $f_{\mathrm{H} \alpha, \mathrm{p}^{\ddagger}}$ & $\begin{array}{c}v_{\mathrm{p}} \\
\left(\mathrm{km} \mathrm{s}^{-1}\right)\end{array}$ & $\begin{array}{c}\text { FWHM } \\
\left(\mathrm{km} \mathrm{s}^{-1}\right)\end{array}$ & $f_{[\mathrm{N} \mathrm{II}] 6583, \mathrm{p}^{\ddagger}}$ & $f_{[\mathrm{S} \mathrm{II}] 6717, \mathrm{p}^{\ddagger}}$ & $f_{[\mathrm{S} \mathrm{II}] 6731, \mathrm{p}^{\ddagger}}$ & $\begin{array}{c}N_{\mathrm{e}, \mathrm{p}} \\
\left(\mathrm{cm}^{-3}\right)\end{array}$ \\
\hline R1 & 4.5 & 35 & 44 & $252 \pm 11$ & $219 \pm 19$ & 24 & 4.2 & 4.8 & $890 \pm 10$ \\
R2 & 3.4 & 37 & 65 & $247 \pm 11$ & $203 \pm 21$ & 33 & 6.7 & 7.6 & $910 \pm 10$ \\
R3 & 12.7 & 65 & 12 & $317 \pm 14$ & $279 \pm 15$ & 6.4 & 0.70 & 0.67 & $520 \pm 50$ \\
R4 & 10.5 & 49 & 9.8 & $240 \pm 15$ & $268 \pm 16$ & 4.6 & 0.51 & 0.40 & $130 \pm 50$ \\
R5 & 11.4 & 51 & 12 & $226 \pm 13$ & $221 \pm 19$ & 6.9 & 1.2 & 1.2 & $740 \pm 40$ \\
R6 & 9.8 & 54 & 8.6 & $227 \pm 15$ & $221 \pm 19$ & 4.1 & 0.44 & 0.47 & $750 \pm 130$ \\
R7 & 10.4 & 38 & 5.6 & $213 \pm 14$ & $312 \pm 14$ & 3.0 & 0.50 & 0.44 & $310 \pm 40$ \\
R8 & 15.4 & 36 & 15 & $234 \pm 13$ & $240 \pm 18$ & 8.1 & 1.3 & 1.5 & $940 \pm 20$ \\
\hline
\end{tabular}

* The intensity-weighted mean polarization degree of the $\mathrm{H} \alpha$ emission.

$\dagger$ The intensity-weighted mean position angle of the polarization vector of the $\mathrm{H} \alpha$ emission.

$\ddagger$ The polarized emission line flux in units of $10^{-16} \mathrm{erg} \mathrm{s}^{-1} \mathrm{~cm}^{-2}$.

with respect to the total spectra (see also Figures $2-5$ ). Also, the polarized $\mathrm{H} \alpha$ lines have radial velocities higher than the systemic velocity of M82 $\left(v_{\text {sys }}=203 \mathrm{~km} \mathrm{~s}^{-1}\right.$; Shopbell \& Bland-Hawthorn 1998; Götz et al. 1990). The velocity excess, $v_{\mathrm{p}}-v_{\text {sys }}$, ranges from $\sim 10 \mathrm{~km} \mathrm{~s}^{-1}$ to $\sim 100$ $\mathrm{km} \mathrm{s}^{-1}$. In the regions close to the nucleus (R1 and R2) $v_{\mathrm{p}}-v_{\mathrm{sys}} \approx 50 \mathrm{~km} \mathrm{~s}^{-1}$, increasing to $\approx 100 \mathrm{~km} \mathrm{~s}^{-1}$ in R3. Note that R3 is in the northwest part of the superwind of M82. In the southeast regions ( $\mathrm{R} 4-\mathrm{R} 8)$, whose distances from the nucleus are $0.7-1 \mathrm{kpc}$, the polarized $\mathrm{H} \alpha$ lines have almost constant velocities, and $v_{\mathrm{p}}-v_{\text {sys }} \sim 10 \mathrm{~km} \mathrm{~s}^{-1}$. In contrast, the $\mathrm{H} \alpha$ lines of the total light are blueshifted relative to $v_{\text {sys }}$ in $\mathrm{R} 4-\mathrm{R} 8$, which is consistent with previous studies (Shopbell \& Bland-Hawthorn 1998; Greve 2004).

The emission line intensity ratios $\left[\begin{array}{ll}\mathrm{N} & \mathrm{II}\end{array}\right] / \mathrm{H} \alpha$ and $[\mathrm{S} \mathrm{II}](\lambda 6717+\lambda 6731) / \mathrm{H} \alpha$ for the polarized light are similar to those of the nuclear region. Figure 7 shows a plot of the ratios for the total light and the polarized light, together with the data taken around the nucleus (Westmoquette et al. 2009). The data for the polarized light are distributed in and around the range of the nuclear data. The data for the total light, however, are distributed well outside the nuclear data (Figure 7).

The emission line intensity ratios $\left[\begin{array}{ll}\mathrm{S} & \mathrm{II}\end{array}\right] \lambda 6731 /$ [S II] $\lambda 6717$ of the polarized spectra are higher than those of the total light in all regions except R4 (Figures 4 and $5)$. Figure 8 shows the $N_{\mathrm{e}}$ derived from the [S II] line ratios. The electron densities $N_{\mathrm{e}, \mathrm{t}}$ obtained from the [S II] line ratios of the total light are $\approx 400 \mathrm{~cm}^{-3}$ at $\sim 0.2 \mathrm{kpc}$ from the nucleus and decrease to $<300 \mathrm{~cm}^{-3}$ at $>0.5$ kpc (Figure 8). In contrast, the electron densities $N_{\mathrm{e}, \mathrm{p}}$ measured in the polarized spectra are almost constant $(\approx$ $500-1000 \mathrm{~cm}^{-3}$ ), except in the two low-density regions (R4 and R7). This indicates that scattered light originates from a high-density region. The electron density of the nucleus of M82 is $\approx 1000 \mathrm{~cm}^{-3}$ (Westmoquette et al. 2009). The $N_{\mathrm{e}, \mathrm{p}}$ we derived are slightly smaller than, but almost consistent with, the nuclear $N_{\mathrm{e}}$.

The foregoing polarized light characteristics (polarization angles, emission line ratios, and $N_{\text {e,p }}$ measured in the polarized light) are consistent with the idea that the polarized light is nuclear light scattered by the dust in the 


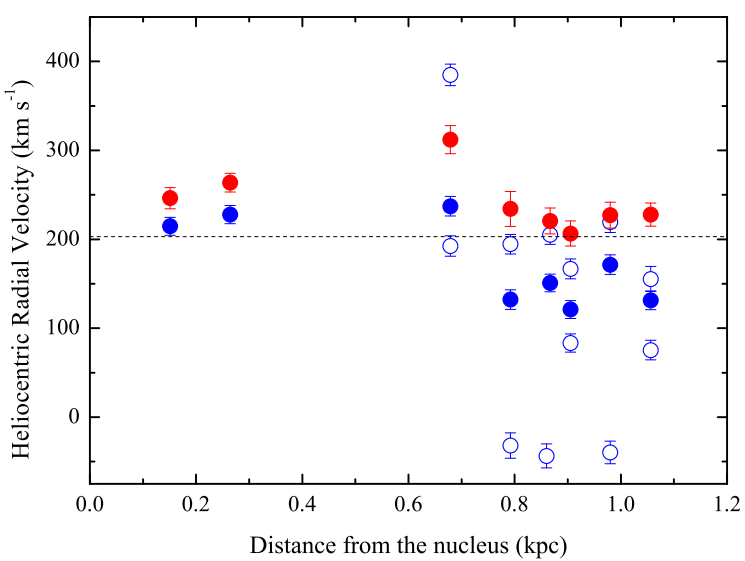

Fig. 6. Position-velocity diagram of the $\mathrm{H} \alpha$ emission of the superwind of M82. The filled red and blue circles indicate the heliocentric radial velocities of the polarized $\mathrm{H} \alpha$ emission and the intensity-averaged velocities of the $\mathrm{H} \alpha$ emission for the total light, respectively. The profiles of the total light emission lines in the outer regions (R3-R8) are fitted with double Gaussian functions, and the velocities of the split components are then plotted as open blue circles. The dotted line represents the systemic velocity of M82.

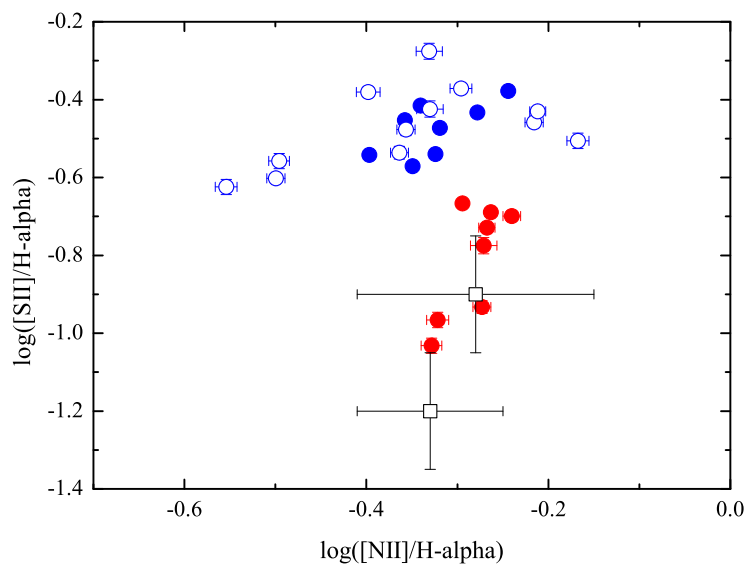

Fig. 7. The emission line intensity ratios of the polarized light and the total light $[\mathrm{S} \quad \mathrm{II}](6717+6731) / \mathrm{H} \alpha$ vs. $[\mathrm{N}$ II $] 6584 / \mathrm{H} \alpha$. The filled red and blue circles indicate the data for the polarized light and the total light, respectively. The open blue circles represent the data for the split components of the total light. The open squares show the data in an area of $\approx 50 \times 100 \mathrm{pc}$ around the nucleus of M82, measured by integral field spectroscopy (Westmoquette et al. 2009). The upper and lower squares represent the data for the narrow component and the broad component, respectively ("C1" and "C2" in Westmoquette et al. (2009)). The error bars associated with the squares show the distribution of the data over the measured region.

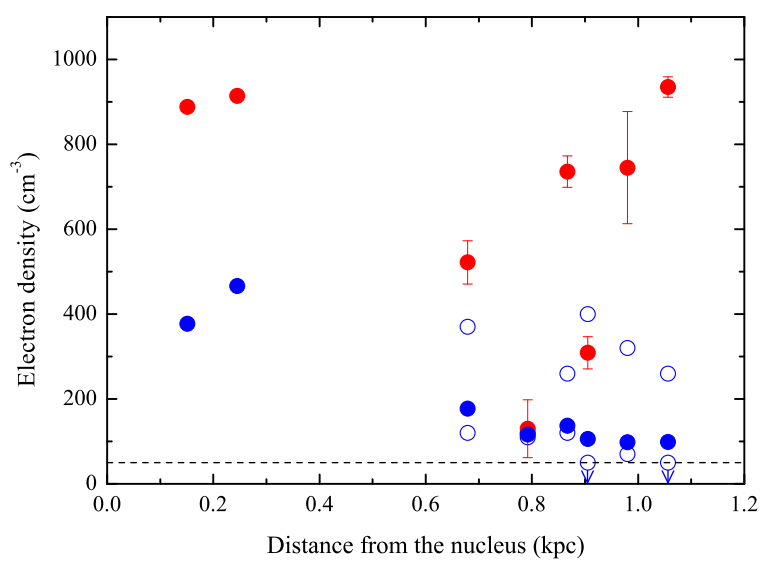

Fig. 8. Electron density derived from the [S II] emission lines of M82. The filled red and blue circles indicate the data for the polarized light and total light, respectively. The open blue circles represent the data for the split components of the total light. The dashed line marks the lower limit of electron density that can be derived from the intensity ratios of the [S II] emission lines.

superwind of M82. In addition, we discovered that the radial velocities of the polarized emission lines are systematically greater than the velocity $v_{\text {sys }}$ of the galaxy. The dust grains that scatter the nuclear light can be inferred to move outward from the nucleus. Accordingly, our research strongly suggests that dust grains entrained by the superwind are transported outward from the disk by the wind and scatter the nuclear light.

\section{Outflow velocity of the dust wind}

We attempt to derive the true outflow velocity of the dust grains in the superwind of M82 using the polarized spectra. Assuming that the radial velocities of the polarized emission lines reflect the motion of the dust grains with respect to the nucleus, we construct a hollow biconical outflow model for the dust outflow of M82 (Figure 9). Since forward scattering is much more efficient for scattering by ordinary interstellar dust grains (van de Hulst 1957), we ignored the backscattering component from the far sides of the outflow cones.

The idea of the model is as follows. Dust grains are flowing outward from the galaxy disk along the walls of the hollow cones, with outflow velocity $v_{\mathrm{d}}$. The radius of the base of the outflow cone is $b \mathrm{kpc}$. The dust grains act as moving mirrors, scattering the light from the nucleus. The opening angle of the outflow is $2 \times \theta$, and the angle of inclination of the axis of the outflow cone is $i$ (Figure 9). Although $i$ is generally different from the angle of inclination of the galaxy disk (i.e., the axis of the cone may be tilted with respect to the rotational axis of the galaxy), the difference between the two values should be small, as suggested by the ionized gas wind morphology (Shopbell \& 
Bland-Hawthorn 1998; Ohyama et al. 2002; Greve 2004). Hence we consider $i$ to be effectively the same as the inclination of the galaxy. In the following calculation, the sign of $i$ is negative on the northwestern side of the galaxy and positive on the southeastern side. When the dust grains are observed at a projected height $h$ above the nucleus, the distance $z$ between the observed dust grains and the galaxy disk ("true height") is expressed as follows:

$$
z=\frac{h+b \tan (i) \cos (i)}{1-\tan (\theta) \tan (i)} .
$$

The distance $r_{\mathrm{d}}$ between the position of the dust grains projected onto the galaxy disk and the nucleus (i.e., the radial distance within the galaxy disk) is then $r_{\mathrm{d}}=$ $z \tan (\theta)+b$. Using these values, we can express the angle $\psi$ between the outflow velocity $v_{\mathrm{d}}$ and the line connecting the observed flow region and the nucleus as

$$
\psi=\pi / 2-\theta-\tan ^{-1}\left(\frac{z}{r_{\mathrm{d}}}\right) .
$$

The dust grains are receding from the nucleus with a velocity $v_{\mathrm{d}} \cos (\psi)$ (red arrows in Figure 9), and the scattered emission lines are redshifted with this velocity. In contrast, the velocity component of the grains along the line of sight is $v_{\mathrm{d}} \sin (\theta+i)$ (blue arrows in Figure 9), and hence the scattered emission lines, are blueshifted with this velocity, relative to the systemic motion of the galaxy. As a result, the observed radial velocity $v_{\mathrm{p}}$ of the scattered emission lines will be $v_{\mathrm{p}}=v_{\mathrm{d}} \cos (\psi)-\left\{v_{\mathrm{d}} \sin (\theta+i)-v_{\text {sys }}\right\}$. In this simple model, we can thus derive the dust outflow velocity $v_{\mathrm{d}}$ as follows:

$$
v_{\mathrm{d}}=\frac{v_{\mathrm{p}}-v_{\mathrm{sys}}}{\cos (\psi)-\sin (\theta+i)},
$$

where $v_{\mathrm{p}}$ and $v_{\mathrm{sys}}\left(=203 \mathrm{~km} \mathrm{~s}^{-1}\right.$; Götz et al. 1990) are the radial velocity of the polarized emission lines and the systemic velocity of M82, respectively.

To calculate $v_{\mathrm{d}}$, we make the following assumptions about the parameters, $b, \theta$, and $i$. The dust can be reasonably expected to be entrained from the galaxy disk outside the main hot outflow. The radius of the base of the dust outflow is then assumed to be the same as the radius of the central starburst, so that $b \approx 0.3 \mathrm{kpc}$ (Greve 2004). The opening angle $2 \theta$ and the angle of inclination $i$ of the dust outflow are hardly determined only by our observations. We examine two cases for $\theta$ : a narrow-angle case and a wide-angle case. In the narrow-angle case, we assume that the dust grains move in the same direction as the ionized gas. In this case, we adopt the value $2 \theta=25^{\circ}$, which was derived by Greve (2004). If the dust flow is associated with molecular gas, $\theta$ may be much wider than the ionized gas flow (the wide-angle case). When the dust grains are associated with the molecular outflow identified by Walter et al. (2002), $2 \theta \approx 50^{\circ}$. Ohyama et al. (2002) suggested a wider opening angle for the dust outflow in or near the galaxy disk $\left(2 \theta>90^{\circ}\right)$ using an optical extinction map (see also Ichikawa et al. 1994). However, the dust scattering the nuclear light is very far away from the disk according to the imaging polarimetry maps (Scarrott

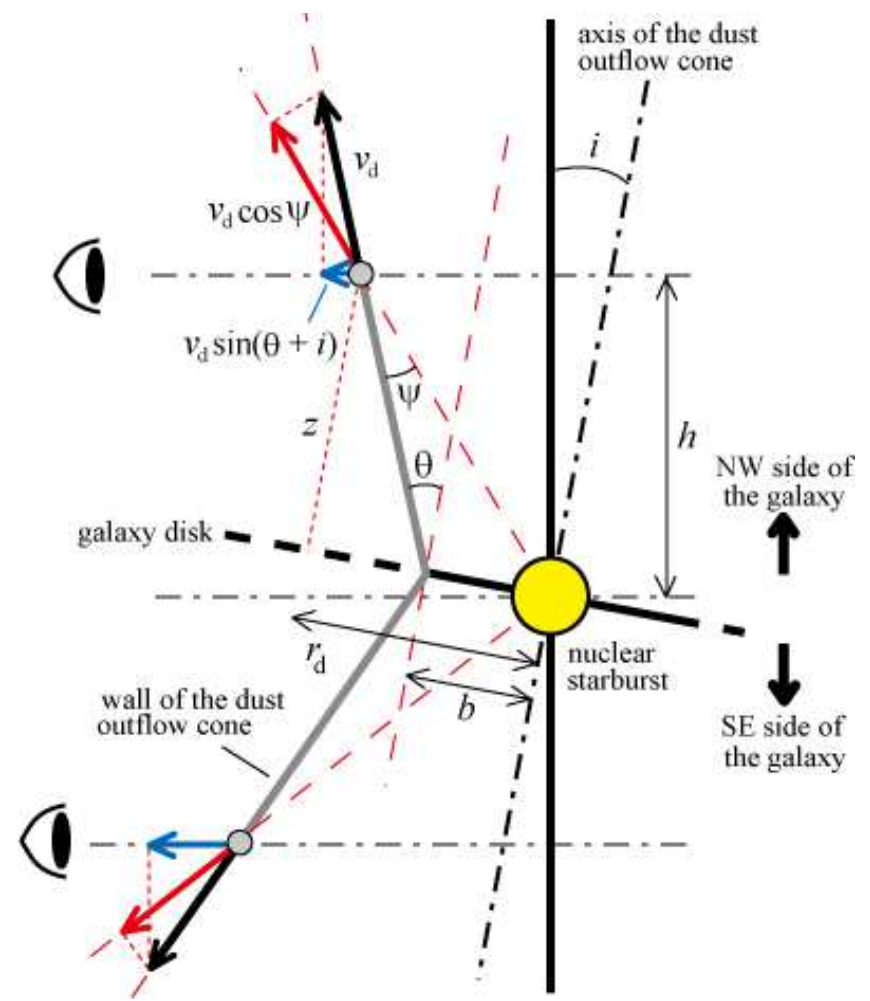

Fig. 9. A schematic diagram of the M82 dust wind. The dust grains in the galaxy disk are assumed to be entrained by the superwind expelled from a circumnuclear circular region whose radius in the disk is $b$. The entrained dust flows along the walls of a cone whose opening angle is $2 \times \theta$. The angle of inclination of the axis of the dust flow cone is $i$. The dust grains in the wind reflect the nuclear emission, acting as mirrors moving at velocity $v_{\mathrm{d}} \cos \psi$ with respect to the nucleus, where $v_{\mathrm{d}}$ is the outflow velocity of the dust.

et al. 1991). Hence the wider-angle dust flows near the disk plane would not contribute to the polarized light we detected here. Thus, we adopt the value $2 \theta=50^{\circ}$ in the wide-angle case. Finally, we assume that $i=15^{\circ}$, which was derived by Greve (2004), for both cases.

We find that the estimated dust outflow velocities based on the above simple model are much less than the escape velocity of M82. In Figure 10, the dust outflow velocities $v_{\mathrm{d}}$ of all regions are plotted against the height $z$ measured from the galaxy disk for the above two cases. The dust outflow velocity $v_{\mathrm{d}}$ decreases almost monotonically with $z$ and reaches a minimum value of $\sim 20-30 \mathrm{~km} \mathrm{~s}^{-1}$ at approximately 1-1.2 kpc in both cases. Martin (1998) estimated the escape velocity of M82 using the $\mathrm{H}$ I velocity field and found that $v_{\mathrm{esc}} \approx 170 \mathrm{~km} \mathrm{~s}^{-1}$ at $1.5 \mathrm{kpc}$ from the nucleus (Martin 1998). The present estimate for $v_{\mathrm{d}}$ is much less than this value. Some indication of an upturn in $v_{\mathrm{d}}$ for $z \gtrsim 1.2 \mathrm{kpc}$ is observed (see Figure 10), but whether this trend reflects a global acceleration of the dust grains at high altitudes above the disk is not clear, owing to the lack of data beyond $z \sim 1.5 \mathrm{kpc}$.

We fit a constant deceleration model to the dust velocity field in the M82 superwind and estimate the dust recycling timescale. Assuming that the dust grains are 


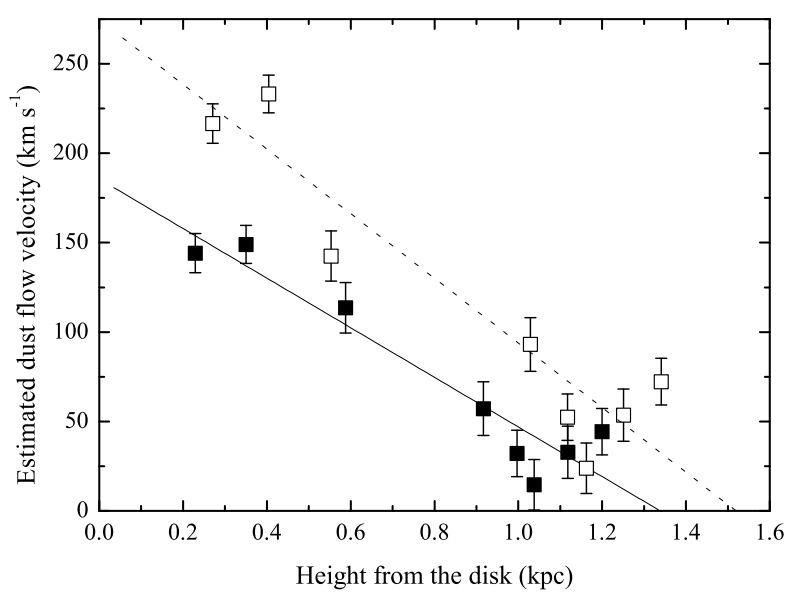

Fig. 10. Estimated dust outflow velocity $v_{\mathrm{d}}$ of M82. The filled squares and open squares represent the data derived for the narrow-angle case and the wide-angle case, respectively. The horizontal axis denotes the height measured from the galaxy disk ( $z$ in Figure 9). The solid and dotted lines represent best-fit curves of constant deceleration models (see text) for the narrow-angle case and the wide-angle case, respectively.

entrained by the superwind at an initial velocity $v_{\mathrm{d}, 0}$ and are decelerated at a constant rate $d_{\mathrm{r}}$, we fit linear functions $v_{\mathrm{d}}(z)=v_{\mathrm{d}, 0}+d_{\mathrm{r}} z$ for the two cases and find $\left(v_{\mathrm{d}, 0} \mathrm{~km} \mathrm{~s}^{-1}, d_{\mathrm{r}} \mathrm{km} \mathrm{s}^{-1} \mathrm{kpc}^{-1}\right)=(186,-139)$ for the narrow-angle case and $(268,-176)$ for the wide-angle case. We can then calculate the distance $z(t)$ (in kpc) attained by the dust grains at time $t$ (in Myr) from $z(t)=v_{\mathrm{d}, 0} / d_{\mathrm{r}} \cdot\left\{1-\exp \left(-1.1 \times 10^{-3} \cdot d_{\mathrm{r}} t\right)\right\}$, since the grains are entrained from the galaxy disk. In this simple model, the dust grains reach a height of $1 \mathrm{kpc}$ above the disk in $\sim 10^{7} \mathrm{yr}$ in both cases. If the deceleration rate $d_{\mathrm{r}}$ is constant, $v_{\mathrm{d}}$ would equal zero at $\sim 1.5 \mathrm{kpc}$ above the disk. The free-fall timescale from $1.5 \mathrm{kpc}$ above the disk of M82 to the disk plane is calculated as $2-3 \times 10^{7} \mathrm{yr}$, under the assumption that a mass of $\sim 10^{10} \mathrm{M}_{\odot}$ is confined within $2 \mathrm{kpc}$ from the M82 nucleus (Sofue, Y. 1992). Hence, if the deceleration of the dust flow is monotonic, the dust expelled by the superwind may return to the disk in several times $10^{7}$ yr. Note that this timescale is comparable to that of starburst activity.

\section{Radial extension of the dust outflow of M82}

Our simple dust outflow model suggests that dust grains cannot go beyond $1.5 \mathrm{kpc}$ above the disk of M82. However, a body of evidence indicates that dust actually distributes over a few kpc around M82. The imaging polarimetry observations of Scarrott et al. (1991) showed that the $\mathrm{H} \alpha$ emission is highly polarized even at the edge of the ionized gas flow of M82 (Scarrott et al. 1991). At the tip of the $\mathrm{H} \alpha$ emission region, $\sim 3 \mathrm{kpc}$ from the disk, the polarization degree reaches $30 \%$, which means that a significant amount of dust exists at that altitude. Sub-mm observations have detected dust emission extending more than a few kpc from the disk (Leeuw \& Robson 2009). An $8-\mu \mathrm{m}$ image taken with the Spitzer infrared satellite revealed a complex filamentary dust structure extending $3 \mathrm{kpc}$ from the disk (Engelbracht et al. 2006). Recently, very extended extraplanar cold dust emission in M82 was discovered via mid- and far-IR observations (Kaneda et al. 2010; Roussel et al. 2010). The UV emission extending to $6 \mathrm{kpc}$ along the minor axis of M82 has also been attributed to dust scattering (Hoopes 2005).

How dust grains are transported to such a high altitude remains a mystery. If the dust grains originate from the galaxy disk, additional mechanisms for their outward acceleration (such as radiation pressure from the starburst or further mechanical acceleration by the wind) are needed to explain such an extended dust distribution. Ferrara et al. (1991) calculated the effect of radiation pressure on the motion of interstellar dust grains. They determined that dust grains are expelled to intergalactic space at several hundred $\mathrm{km} \mathrm{s}^{-1}$ by the radiation pressure of stellar light in our Galaxy and the star-forming galaxy NGC 3198. Their calculation predicted that the velocity of the dust would rapidly increase to $\sim 500 \mathrm{~km} \mathrm{~s}^{-1}$ within 10 Myr from the onset of the flow and then decrease to $\sim 100-200 \mathrm{~km} \mathrm{~s}^{-1}$ in 100 Myr. The dust grains are transported outward to an order of $100 \mathrm{kpc}$ in $100 \mathrm{Myr}$.

The results of Ferrara et al.'s calculation (Ferrara et al. 1991) clearly contradict our observational results for M82. Our observations suggest that $v_{\mathrm{d}}$ decreases with time after the onset of the flow (see Section 5). Note that radiation pressure is highly dependent on the dust grain size $a$. Radiation pressure is proportional to $a^{2}$, whereas gravitational force is proportional to $a^{3}$. Although the grain size distribution and spatial distribution of the extraplanar dust of M82 are not known, dust components traced by mid-IR observations (Engelbracht et al. 2006; Bierão et al. 2008; Kaneda et al. 2010) should consist primarily of small PAH dust particles. These small dust particles may be transported very far away from the disk by radiation pressure and form a highly protracted structure. Dust particles involved in optical scattering, however, are much larger and heavier than PAH dust; submicron-sized grains are mostly responsible for optical scattering $(2 \pi a / \lambda \sim 1$; e.g., van de Hulst 1957). Thus, the discrepancy between mid-IR dust extension and optical scattering dust kinematics is possibly reconciled by the size difference between the grains responsible for each emission.

Note, however, that the $\mathrm{H} \alpha$ emission is scattered even at the tip of the bright $\mathrm{H} \alpha$ filaments (Scarrott et al. 1991), indicating that optical scattering dust is still present at this distance. At least two possible explanations exist for this finding: 1) The origin of the outer dust is the same as that of the inner dust, and the grains are accelerated by some mechanism(s) at $\geq 1 \mathrm{kpc}$. 2) The origin of the outer dust is different from that of the inner dust. Some indication of dust acceleration is observed at around $1.2 \mathrm{kpc}$ 
from the disk, since a velocity upturn appears in the velocity field we obtained (see the previous section). This may indicate that the dust grains are accelerated outward beyond $1 \mathrm{kpc}$. However, the velocity upturn is not especially significant, since the lack of data points makes it impossible to be certain whether the dust is really accelerated beyond $1 \mathrm{kpc}$. However, the distant dust may be associated with the $\mathrm{H}$ I stream traversing the disk of M82 (Yun et al. 1993). This stream is part of a giant H I cloud complex extending across the M81/M82 group, and possibly formed by a galaxy-galaxy interaction between M82 and M81 (Appleton, Davies \& Stephenson 1981; Chynoweth et al. 2008). Recently, Roussel et al. (2010) detected a protracted cold dust emission around M82 via far-IR (250 $\mu \mathrm{m}, 350 \mu \mathrm{m}$ and $500 \mu \mathrm{m})$ observation with the Herschel satellite. The cold dust extends to $9 \mathrm{kpc}$ from the center. By comparing their far-IR maps with other wavelength data, they found that the outer part of the cold dust is associated with the $\mathrm{H}$ I stream, while the inner part is dominated by the warm $(\sim 30-50 \mathrm{~K})$ component associated with the superwind (Roussel et al. 2010). In this case, optical scattering dust far away from the disk is provided by this stream, and the dust velocity field in the outer part of the superwind may reflect $\mathrm{H}$ I stream kinematics. To distinguish between the two possibilities listed above, one must trace the motion of the dust toward a higher altitude above the disk.

\section{Comparison with other outflow materials}

We compare the kinematics of the dust and other outflowing materials, including ionized gas, molecular gas, and neutral atomic gas, in the superwind of M82.

The kinematical dissociation between the dust and the warm ionized gas (optical emission line gas) of M82 is very clear. The outflow velocity of the ionized gas increases almost linearly with the distance from the disk at a rate of $300-400 \mathrm{~km} \mathrm{~s}^{-1} \mathrm{kpc}^{-1}$ (Greve 2004). In contrast, the dust outflow decelerates at a rate of $-150 \mathrm{~km} \mathrm{~s}^{-1} \mathrm{kpc}^{-1}$ (see Section 5). The final outflow velocity of the ionized gas in M82 reaches $\sim 500-600 \mathrm{~km} \mathrm{~s}^{-1}$ at $\sim 1 \mathrm{kpc}$ from the disk (Shopbell \& Bland-Hawthorn 1998; Greve 2004), which is much higher than the dust velocity we derived.

The velocity field of the molecular gas in the M82 superwind is also inconsistent with the kinematics of the dust. Several CO observations have detected a molecular gas outflow with velocity $\sim 200 \mathrm{~km} \mathrm{~s}^{-1}$ in M82 (Seaquist \& Clark 2001; Walter et al. 2002). This value is almost the same as the dust velocity near the nucleus $(z<0.4 \mathrm{kpc})$ for both the narrow- and wide-angle cases of our dust outflow model. Hence dust and CO gas may be associated with each other near the nucleus. The spatial coincidence between CO emission and the $450-\mu \mathrm{m}$ emission also supports a spatial association of dust and molecular gas in the central region (Hughes et al. 1994). However, the CO outflow velocities $\left(\sim 200 \mathrm{~km} \mathrm{~s}^{-1}\right)$ were measured at $\sim 1$ kpc from the nucleus, where the dust velocity decreases to less than $100 \mathrm{~km} \mathrm{~s}^{-1}$ (Figure 10). Thus, we can conclude that molecular gas is also kinematically decoupled from the dust at high altitudes in the superwind of M82.

Finally, we compare dust motion with neutral atomic gas motion. Heckman et al. (2000) discovered that Na I absorption lines are systematically blueshifted with respect to the systemic velocities of starburst galaxies (see also Martin et al. 2002, 2005; Rupke et al. 2002, 2005; Schwartz \& Martin 2004). They interpreted this phenomenon as a neutral gas outflow from the starburst region. Using a correlation between the optical absorption coefficient $A_{V}$ and the equivalent width of the $\mathrm{Na} \mathrm{I}$ absorption line, they inferred that dust is associated with neutral atomic gas, which is represented by Na I absorption. The outflow velocity derived from the blueshift of the $\mathrm{Na}$ I lines of M82 is $\approx 80 \mathrm{~km} \mathrm{~s}^{-1}$. This value is significantly smaller than $v_{\mathrm{d}}$ near the nucleus, which may imply that neutral atomic gas is also kinematically decoupled from the dust near the nucleus. However, we should note that Heckman et al. (2000) measured the line-of-sight velocity of $\mathrm{Na}$ I only in the nuclear region. If the outflow were spherically symmetric, the Na I velocity they derived would equal the actual outflow velocity. However, various observations indicate that the superwind of M82 has a bipolar nature. Hence the Na I velocity represents not the extraplanar outflow velocity, but the expansion velocity of the circumnuclear gas in the galactic plane. In other words, currently available data do not allow us to directly compare the kinematics of dust and neutral atomic gas. Deep spectroscopy of $\mathrm{Na}$ I absorption lines at large radii in M82 would help us to study the detailed outflow kinematics of neutral atomic gas and to compare it with dust kinematics.

\section{Conclusion}

We conducted optical spectropolarimetry observations of the starburst superwind of the prototypical starburst galaxy M82 to reveal the motion of the dust entrained by the superwind. The $\mathrm{H} \alpha$ polarization degrees $(\sim 5-15 \%)$ and angles measured by our study are consistent with previous narrowband imaging polarimetry maps. The polarized emission lines are redshifted relative to the systemic motion of the galaxy. The emission line intensity ratios $[\mathrm{N}$ II] $/ \mathrm{H} \alpha$ and $[\mathrm{S}$ II] $/ \mathrm{H} \alpha$ in the polarized light are similar to those of the nuclear star-forming region. The electron densities $N_{\mathrm{e}}$ derived from the polarized [S II] line ratio are much higher than those derived from the total light. These facts strongly suggest that the emission from a nuclear starburst is scattered by dust grains entrained and then transported outward by the starburst superwind. We derived the outflow velocity of the dust grains, $v_{\mathrm{d}}$, using a simple hollow biconical outflow model. The outflow velocity $v_{\mathrm{d}}$ is on the order of a few hundred $\mathrm{km} \mathrm{s}^{-1}$ near the nucleus and decreases monotonically with the distance from the nucleus. The dust motion revealed by this study is substantially slower than the motion of the other components of the superwind (ionized gas and molecular gas). The outflow velocity of the dust is also much less than the escape velocity of M82. In the absence of any additional effective acceleration mechanisms (such as radiation pres- 
sure), the dust expelled by the superwind would fall back into the galaxy disk within several times $10^{7} \mathrm{yr}$.

We are grateful to the Subaru Telescope staff for their kind assistance with the observations. We also thank the anonymous referee for his/her helpful comments. Part of this study was carried out using the facilities of the Astronomical Data Center, National Astronomical Observatory of Japan. This research made use of NASA's Astrophysics Data System Abstract Service. This work was financially supported in part by the Japan Society for the Promotion of Science (Grant-in-Aid for Scientific Research No. 18340055) and the Ministry of Education, Culture, Sports, Science \& Technology, Japan (Grantin-Aid for Scientific Research on Priority Areas No. 19047003).

\section{References}

Alton, P. B., Davies, J. I. \& Bianchi, S. 1999, A\&A, 343, 51

Appleton, P. N., Davies, R. D. \& Stephenson, R. J. 1981, MNRAS, 195, 327

Bierão, P. et al. 2008, ApJ, 676, 304

Bingham, R. G. et al. 1976, Nature, 259, 463

Bland, J. \& Tully, R. B. 1988, Nature, 334, 43

Bregman, J. N., Schulman, E. \& Tomisaka, K. 1995, ApJ, 439, 155

Chevalier, R. A. \& Clegg, A. W. 1985, Nature, 317, 44

Chynoweth, K. M. et al. 2008, AJ, 135, 1983

Engelbracht, C. W. et al. 2006, ApJL, 642, L127

Ferrara, A., Ferrini, F., Franco, J. \& Barsella, B. 1991, ApJ, 381,137

Götz, M., McKeith, C. D., Downes, D. \& Greve, A. 1990, A\&A, 240, 52

Greve, A. 2004, A\&A, 416, 67

Heckman, T. M. et al. 2000, ApJS, 129, 493

Heckman, T. M. 2003, RevMexAA, 17, 47

Hoopes, C. G. et al. 2005, ApJL, 619, L99

Hughes, D. H., Gear, W. K \& Robson, E. I. 1994, MNRAS, 270,641

Ichikawa, T. et al. 1994, ApJ, 433, 645

Kaifu, N., et al. 2000, PASJ, 52, 1

Kaneda et al. 2010, A\&A, 514, A14

Kashikawa, N., et al. 2002, PASJ, 54, 819

Kawabata, K. et al. 2003, SPIE, 4841, 1219

Leeuw, L. L. \& Robson, E. I. 2009, AJ, 137, 517

Martin, C. L. 1998, ApJ, 506, 222

Martin, C. L., Kobulnicky, H. A. \& Heckman, T. M. 2002, ApJ, 574, 663

Martin, C. L. 2005, ApJ, 621, 227

Matsushita, S. et al. 2005, ApJ, 618, 712

Mutchler, M. et al. 2007, PASP, 119, 1

Nakai, N. et al. 1987, PASJ, 39, 685

Ohyama, Y. et al. 2002, PASJ, 54, 891

Ranalli, P., Comastri, A., Origlia, L. \& Maiolino, R. 2008, MNRAS, 386, 1464

Roussel, H. et al. 2010, A\&A, 518, L66

Rupke, D. S., Veilleux, S. \& Sanders, D. B. 2002, ApJ, 570, 588

Rupke, D. S., Veilleux, S. \& Sanders, D. B. 2005, ApJS, 160, 115

Sakai, S. \& Madore, B. F. 1999, ApJ, 526, 599
Scarrott, S. M., Eaton, N. \& Axon, D. J. 1991, MNRAS, 252, 12

Schmidt, G. D., Angel, J. R. P. \& Comwell, R. H. 1976, ApJ, 206, 888

Schwartz, C. M. \& Martin, C. L. 2004, ApJ, 610, 201

Seaquist, E. R. \& Clark, J. 2001, ApJ, 552, 133

Seaquist, E. R., Lee, S. W. \& Moriarty-Schieven, G. H. 2006, ApJ, 638, 148

Shopbell, P. L. \& Bland-Hawthorn, J. 1998, ApJ, 493, 129

Sofue, Y. et al. 1992, ApJ, 395, 126

Strickland, D. K. \& Stevens, I. R. 2000, MNRAS, 314, 511

Strickland, D. K. \& Heckman, T. M. 2007, ApJ, 658, 258

Taylor, C. L., Walter, F. \& Yun, M. S. 2001, ApJL, 562, L43

Telesco, C. M. et al. 1991, ApJ, 369, 135

Thuma et al. 2000, A\&A, 358, 65

Tinbergen, J. 1996, Astronomical Polarimetry (New York: Cambridge Univ. Press)

Tsuru, T. G. et al. 2007, PASJ, 59, 269

van de Hulst, H. C. 1957, Light Scattering by Small Particles (New York: J. Wiley \& Sons)

Veilleux, S., Cecil, G. \& Bland-Hawthorn, J. 2005, ARA\&A, 43, 769

Visvanathan, N. \& Sandage, A. R. 1972, ApJ, 176, 57

Walter, F., Weiß, A. \& Scoville, N. Z. 2002, ApJL, 580, L21

Weiß, A, Walter, F. \& Scoville, N. Z. 2005, A\&A, 458, 533

Westmoquette, W. S. et al. 2009, ApJ, 706, 1571

Yun, M. S. et al. 1993, ApJL, 411, L17 